\title{
INJECTIVES IN FINITELY GENERATED UNIVERSAL HORN CLASSES
}

\author{
MICHAEL H. ALBERT ${ }^{1}$ AND ROSS WILLARD ${ }^{2}$
}

\begin{abstract}
Let $\mathbf{K}$ be a finite set of finite structures. We give a syntactic characterization of the property: every element of $\mathbf{K}$ is injective in $\operatorname{ISP}(\mathbf{K})$. We use this result to establish that $\mathscr{A}$ is injective in $\operatorname{ISP}(\mathscr{A})$ for every two-element algebra $\mathscr{A}$.
\end{abstract}

§0. Introduction. Let $\mathbf{K}$ be a finite set of finite structures for a first-order language. In this paper we give a syntactic characterization (Theorem 4 ) of the property that each member of $\mathbf{K}$ is injective in $\mathbf{I S P}(\mathbf{K})$, the universal Horn class generated by $\mathbf{K}$. We then show that $\mathbf{K}=\{\mathscr{A}\}$ has this property for every two-element algebra $\mathscr{A}$.

This paper was motivated by the following question: for which two-element algebras $\mathscr{A}$ does $\operatorname{ISP}(\mathscr{A})$ have the amalgamation property? The property stated above is stronger than the amalgamation property, so the answer is: for every twoelement algebra.

Model companions appear in Lemma 1. For their definition and elementary properties see [8], but note that we replace a theory by its class of models. Theorem 9 rests on E. Post's classification [9] of all two-valued clones of operations, a summary of which appears in [7].

The results of $\S 1$ are joint work of the authors, the individual contributions being inextricably combined but of equal magnitude. $\$ 2$ is due to R. Willard, and the details of $\$ 3$ were also worked out by R. Willard based on an explanation of the results in [1] given by $\mathrm{M}$. H. Albert.

§1. The syntactic characterization. Let $\mathbf{M}$ be a class of structures for some firstorder language. The classes $\mathbf{I}(\mathbf{M}), \mathbf{S}(\mathbf{M}), \mathbf{P}(\mathbf{M})$ and $\mathbf{P}_{\text {fin }}(\mathbf{M})$ are the closures of $\mathbf{M}$ under isomorphism, substructures, products, and finite products respectively. $\mathbf{M}_{\mathrm{fin}}$ is the class of all finite members of $\mathbf{M}$. Arrows and hooked arrows between members of $\mathbf{M}$ denote homomorphisms and embeddings respectively. A member $\mathscr{D}$ of $\mathbf{M}$ is injective in $\mathbf{M}$ if every diagram 1(a) in $\mathbf{M}$ can be completed to a commuting diagram 1(b).

Received April 28, 1986; revised October 17, 1986.

${ }^{1}$ Research supported by an NSERC postdoctoral fellowship.

${ }^{2}$ Research supported by an Ontario graduate scholarship. 


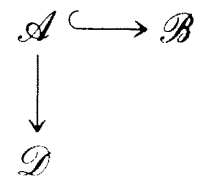

1(a)

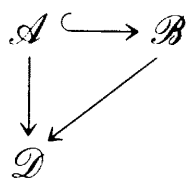

1(b)

$\mathbf{M}^{\text {inj }}$ denotes the class of all members of $\mathbf{M}$ which are injective in $\mathbf{M}$. We say that $\mathbf{M}$ has enough injectives (EI) if $\mathbf{M} \subseteq \mathbf{I S}\left(\mathbf{M}^{\mathrm{inj}}\right)$, and that $\mathbf{M}$ has the transferability property (TP) if every diagram 2(a) in $\mathbf{M}$ can be completed in $\mathbf{M}$ to a commuting diagram 2(b).

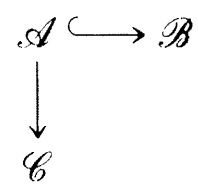

2(a)

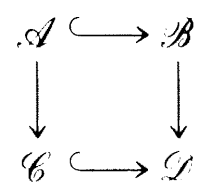

2(b)

Remark. EI implies TP, and $\mathbf{P}\left(\mathbf{M}^{\mathrm{inj}}\right) \cap \mathbf{M}=\mathbf{M}^{\mathrm{inj}}$.

A first-order formula is existential-positive $\left(\exists^{+}\right)$if it is both existential and positive; likewise open-positive $\left(\mathrm{O}^{+}\right)$. A \&at formula is a conjunction of atomic formulas; an $\exists \&$ at formula is one of the form $\exists \vec{y} \phi(\vec{y})$ where $\phi$ is \&at. We say that $\mathbf{M}$ has the property $\exists^{+} \equiv O^{+}$if every $\exists^{+}$formula (in the language of $\mathbf{M}$ ) is equivalent modulo $\mathbf{M}$ to an $O^{+}$formula; likewise $\exists \&$ at $\equiv \&$ at.

Remark. $\exists \&$ at $\equiv$ \&at implies $\exists^{+} \equiv O^{+}$; if $\mathbf{P}_{\text {fin }}(\mathbf{M}) \subseteq \mathbf{I S}(\mathbf{M})$, then $\mathbf{M}$ has $\exists^{+} \equiv O^{+}$ iff it has $\exists \&$ at $\equiv \&$ at.

A member $\mathscr{A}$ of $\mathbf{M}$ is algebraically closed in $\mathbf{M}$ if for every $\mathscr{B} \in \mathbf{M}$, every embedding $\alpha: \mathscr{A} \hookrightarrow \mathscr{B}$, every $\exists^{+}$formula $\phi(\vec{x})^{3}$ and all $\vec{a} \in \mathscr{A}, \mathscr{B} \vDash \phi(\alpha \vec{a})$ implies $\mathscr{A} \vDash \phi(\vec{a})$. The class of all members of $\mathbf{M}$ which are algebraically closed in $\mathbf{M}$ is denoted $\mathbf{M}^{\text {ac }}$.

We first establish some connections between $\mathbf{M}^{\mathrm{inj}}, \mathbf{M}^{\mathrm{ac}}, \exists^{+} \equiv O^{+}$and TP.

LEMMA 1. Let $\mathbf{M}$ be a universal class.

a) If there is a class $\mathbf{N}$ such that $\mathbf{M}=\mathbf{I S}(\mathbf{N})$ and $\mathbf{N}$ has $\exists^{+} \equiv O^{+}$, then $\mathbf{M}$ has TP.

b) The converse is true if $\mathbf{M}$ has a model companion.

ProOF. a) This is a consequence of Theorem 2.1(c) of [2]; however, we prove it directly. Suppose that we have the following diagram in $\mathbf{M}$ :

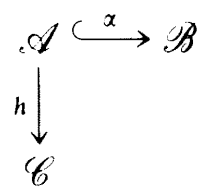

Choose $\beta: \mathscr{B} \hookrightarrow \mathscr{B}^{\prime} \in \mathbf{N}$ and $\gamma: \mathscr{C} \hookrightarrow \mathscr{C}^{\prime} \in \mathbf{N}$. Suppose that $\phi(\vec{x}, \vec{y})$ is an $O^{+}$formula, $\vec{a}$ $\in \mathscr{A}, \vec{b} \in \mathscr{B}$ and $\mathscr{B} \models \phi(\alpha \vec{a}, \vec{b})$. Pick an $O^{+}$formula $\psi(\vec{x})$ such that $\mathbf{N} \models \exists \vec{y} \phi \leftrightarrow \psi$.

\footnotetext{
${ }^{3}$ In order to avoid annoying exceptions, we reserve the symbol $\vec{x}$ (but not $\vec{y}$ ) for nonempty tuples of variables, i.e. n-tuples for $n \geq 1$. Otherwise, our notation is standard.
} 
Then $\mathscr{B}^{\prime} \vDash \exists \vec{y} \phi(\beta \alpha \vec{a}, \vec{y})$, so $\mathscr{B}^{\prime} \models \psi(\beta \alpha \vec{a}), \mathscr{A} \models \psi(\vec{a})$ and $\mathscr{C}^{\prime} \models \psi(\gamma h \vec{a})$; hence $\mathscr{C}^{\prime} \models$ $\exists \vec{y} \phi(\gamma h \vec{a}, \vec{y})$. It now follows by compactness that there is an elementary extension $\mathscr{D}$ of $\mathscr{C}^{\prime}$ and a homomorphism $g: \mathscr{B} \rightarrow \mathscr{D}$ such that $g \circ \alpha=($ incl $\circ \gamma) \circ h$, as desired.

b) Let $\mathbf{N}$ be the model companion of $\mathbf{M}$. All we need to know is that $\mathbf{N}$ is elementary, $\mathbf{N} \subseteq \mathbf{M}^{\mathrm{ac}}$, and $\mathbf{M}=\mathbf{I S}(\mathbf{N})$. Let $\phi(\vec{x})$ be an $\exists^{+}$formula. If $\mathbf{N} \models \neg \phi(\vec{x})$ then clearly $\mathbf{N} \vDash \phi(\vec{x}) \leftrightarrow \psi(\vec{x}, \vec{y})$, where $\psi(\vec{x}, \vec{y})$ is the matrix of $\phi(\vec{x})$. Otherwise we use the following variation of Lemma 3.2.1 in [6]: if $\mathbf{N}$ is elementary and $\phi(\vec{x})$ is a formula such that $\mathbf{N} \# \neg \phi(\vec{x})$, then $\phi$ is equivalent modulo $\mathbf{N}$ to an $O^{+}$formula iff for all $\mathscr{A}, \mathscr{B} \in \mathbf{N}$, all $\vec{a} \in \mathscr{A}$, and every homomorphism $h$ from $\langle\vec{a}\rangle(=$ the substructure of $\mathscr{A}$ generated by $\vec{a})$ to $\mathscr{B}, \mathscr{A} \models \phi(\vec{a})$ implies $\mathscr{B} \models \phi(h \vec{a})$.

Suppose that in our case we have the diagram below, with $\mathscr{A}, \mathscr{B} \in \mathbf{N}$ :

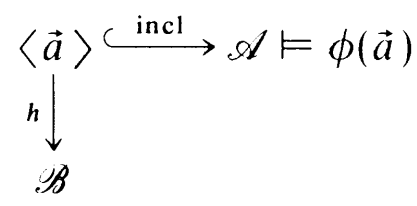

Since $\mathbf{M}$ has TP there are $\mathscr{C} \in \mathbf{M}, \alpha: \mathscr{B} \hookrightarrow \mathscr{C}$, and $g: \mathscr{A} \rightarrow \mathscr{C}$ such that $g \circ$ incl $=\alpha \circ h$. Then $\mathscr{C} \vDash \phi(g \vec{a})$, i.e., $\mathscr{C} \vDash \phi(\alpha h \vec{a})$ and hence $\mathscr{B} \vDash \phi(h \vec{a})$ as desired, since $\phi$ is $\exists^{+}$and $\mathscr{B} \in \mathbf{M}^{\text {ac }}$.

LeMmA 2. Let $\mathbf{M}$ be a universal class and let $\mathbf{N}$ be a class such that $\mathbf{M}=\mathbf{I S}(\mathbf{N})$ and $\mathbf{N}$ has $\exists^{+} \equiv O^{+}$. Then

a) $\mathbf{N} \subseteq \mathbf{M}^{\text {ac }}$, and

b) $\mathbf{M}^{\text {ac }}$ has $\exists^{+} \equiv O^{+}$.

Proof. a) Let $\mathscr{A} \in \mathbf{N}$. Given $\alpha: \mathscr{A} \hookrightarrow \mathscr{B} \in \mathbf{M}$, find $\beta: \mathscr{B} \hookrightarrow \mathscr{C} \in \mathbf{N}$. If $\phi(\vec{x})$ is $\exists^{+}, \vec{a}$ $\in \mathscr{A}$ and $\mathscr{B} \models \phi(\alpha \vec{a})$, then $\mathscr{C} \models \phi(\beta \alpha \vec{a})$. Clearly $\mathbf{N}^{\text {ac }}=\mathbf{N}$ since $\mathbf{N}$ has $\exists^{+} \equiv O^{+}$; hence $\mathscr{A} \vDash \phi(\vec{a})$.

b) Let $\varphi, \psi$ be $\exists^{+}, O^{+}$formulas respectively such that $\mathbf{N} \vDash \phi \leftrightarrow \psi$. An argument like the previous one yields $\mathbf{M}^{\text {ac }} \models \phi \leftrightarrow \psi$.

LEMMA 3. Let $\mathbf{M}$ be a class of structures.

a) $\mathbf{M}^{\text {inj }} \subseteq \mathbf{M}^{\text {ac }}$.

b) $\left(\mathbf{M}^{\text {ac }}\right)_{\text {fin }} \subseteq \mathbf{M}^{\text {inj }}$ if $\mathbf{M}$ has $T P$.

Proof. a) Let $\mathscr{A} \in \mathbf{M}^{\text {inj }}$. Given $\alpha: \mathscr{A} \hookrightarrow \mathscr{B} \in \mathbf{M}$, use the injectivity of $\mathscr{A}$ in the diagram

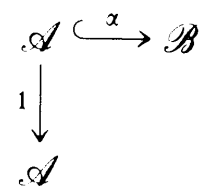

b) Suppose $\mathscr{C} \in\left(\mathbf{M}^{\text {ac }}\right)_{\text {fin }}$ and we have the following diagram in $\mathbf{M}$ :

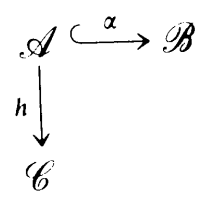

As in the proof of Lemma 1(b), for every $\exists^{+}$formula $\phi(\vec{x})$ and all $\vec{a} \in \mathscr{A}, \mathscr{B} \models \phi(\alpha \vec{a})$ implies $\mathscr{C} \vDash \phi(h \vec{a})$. As in the proof of Lemma 1(a), it follows that there is an 
elementary extension $\mathscr{D}$ of $\mathscr{C}$ and a homomorphism $g: \mathscr{B} \rightarrow \mathscr{D}$ such that $g \circ \alpha$ $=$ incl $\circ h$. But $\mathscr{C}$ is finite, so $\mathscr{D}=\mathscr{C}$ as desired.

We now establish the main result of this section.

THEOREM 4. Let $\mathbf{K}$ be a finite set of finite structures. Then $\mathbf{K} \subseteq \mathbf{I S P}(\mathbf{K})^{\text {inj }}$ iff $\mathbf{K}$ has $\exists \&$ at $\equiv$ \&at.

Proof. $(\Rightarrow)$. Suppose that $\mathbf{K} \subseteq \operatorname{ISP}(\mathbf{K})^{\text {inj. Then }} \mathbf{P}(\mathbf{K}) \subseteq \operatorname{ISP}(\mathbf{K})^{\text {inj }}$ by the first remark of this section, so $\operatorname{ISP}(\mathbf{K})$ has EI and hence TP. ISP $(\mathbf{K})$ has a model companion [5]; thus by Lemmas 1(b) and 2(b), $\mathbf{I S P}(\mathbf{K})^{\text {ac }}$ has $\exists^{+} \equiv O^{+}$and hence $\exists \&$ at $\equiv \&$ at (see the second remark). It remains to note that $\mathbf{K} \subseteq \mathbf{I S P}(\mathbf{K})^{\text {ac }}$ by Lemma 3(a) and the hypothesis.

$(\Leftarrow)$. Suppose that $\mathbf{K}$ has $\exists \&$ at $\equiv$ \&at. Then $\mathbf{P}(\mathbf{K})$ does likewise (since, if $\phi$ and $\psi$ are $\exists \&$ at, then $\forall \vec{x}(\phi(\vec{x}) \leftrightarrow \psi(\vec{x}))$ is equivalent to a Horn sentence and so is preserved under products). Thus ISP(K) has TP by Lemma 1(a), $\mathbf{K} \subseteq\left(\mathbf{I S P}(\mathbf{K})^{\text {ac }}\right)_{\text {fin }}$ by Lemma 2(a), and $\left(\mathbf{I S P}(\mathbf{K})^{\text {ac }}\right)_{\text {fin }} \subseteq \mathbf{I S P}(\mathbf{K})^{\text {inj }}$ by Lemma 3(b).

\$2. A transfer theorem. In this section we reformulate Theorem 4 in the style of clones and use this reformulation to prove a transfer theorem (Corollary 5).

Let $\mathbf{K}$ be a finite set of finite structures for a first order language. $K$ denotes the set of universes of the members of $\mathbf{K}$. We say that $\mathbf{K}$ is regular if no two members share a common universe. We wish to compare regular sets $\mathbf{K}$ and $\mathbf{K}^{\prime}$, not necessarily for the same language, such that $K=K^{\prime}$. The insistence on distinct universes is simply to ensure that there is, in this case, a natural 1-1 correspondence between the structures of $\mathbf{K}$ and those of $\mathbf{K}^{\prime}$.

Let $K$ be any finite set of nonempty finite sets. An $n$-ary operation (relation) on $K$ is a map with domain $K$ which assigns to each $A \in K$ an $n$-ary operation (relation) on $A$. We use $\mathscr{O}_{K}\left(\mathscr{R}_{K}\right)$ to denote the set of all $n$-ary operations on $K, n \geq 0$ (all $n$-ary relations on $K, n \geq 1$ ). We distinguish an operation $f$ from its graph (which is a relation). $\mathscr{L}_{K}$ is the (disjoint) union of $\mathscr{O}_{K}$ and $\mathscr{R}_{K}$.

Let $\mathbf{K}$ be a regular set of structures for some language. $L_{\mathbf{K}}$ is the set of operations and relations on $K$ defined by the symbols in the language; thus $L_{\mathbf{K}} \subseteq \mathscr{L}_{K}$. Conversely, any $L \subseteq \mathscr{L}_{K}$ can be considered as a language (in the obvious way) which has a natural interpretation in $K$. The regular set of $L$-structures so obtained is denoted $K_{L}$.

Fix $K$. For $f \in \mathcal{O}_{K}$, graph $(f)$ denotes the graph of $f$. If $\mathbf{c}$ is a constant (nullary operation) on $K$, then $c$ denotes the unary operation on $K$ defined by $c(x)=\mathbf{c}$. Define $\operatorname{Su}\left(\mathscr{L}_{K}\right)$ to be the set of subsets of $\mathscr{L}_{K}$. We define the maps $C, C_{\text {\&at }}$, and $C_{\text {ᄏ\&at }}$ from $\operatorname{Su}\left(\mathscr{L}_{K}\right)$ to $\operatorname{Su}\left(\mathscr{L}_{K}\right)$ as follows. For $L \subseteq \mathscr{L}_{K}, f \in \mathscr{O}_{K}, p \in \mathscr{R}_{K}$, and constant c $\in \mathscr{O}_{K}$ :

$p \in C_{\& a t}(L)$ iff $p$ is definable in $K_{L}$ by a \&at formula;

$f \in C_{\text {\&at }}(L)$ iff $\operatorname{graph}(f) \in C_{\text {\&at }}(L)$;

$C_{\exists \& a t}$ is defined similarly;

$p \in C(L)$ iff $p \in C_{\text {\&at }}(L)$;

$f \in C(L)$ iff $f$ is definable in $K_{L}$ by an $L$-term, provided that the arity of $f$ is not zero;

$\mathbf{c} \in C(L)$ iff $c(x) \in C(L)$.

It is straightforward to show that these maps are monotone increasing, $L \subseteq C(L)$ $\subseteq C_{\text {\&at }}(L) \subseteq C_{\text {ヨ\&at }}(L)$ for all $L \subseteq \mathscr{L}_{K}, C_{\text {ヨ\&at }}$ is idempotent and $C_{\text {\&at }} C=C_{\text {\&at }}$. 
Theorem 4 now has the following reformulation: for any regular $\mathbf{K}, \mathbf{K} \subseteq \mathbf{I S P}(\mathbf{K})^{\text {inj }}$ iff $C_{\text {ヨ\&at }}\left(L_{\mathbf{K}}\right)=C_{\text {\&at }}\left(L_{\mathbf{K}}\right)$. From this we deduce

COROLlaRY 5. Let $\mathbf{K}$ and $\mathbf{K}^{\prime}$ be regular sets of structures (not necessarily for the same language) such that $K=K^{\prime}$. Suppose that $L_{\mathbf{K}} \subseteq C\left(L_{\mathbf{K}^{\prime}}\right)$ and $L_{\mathbf{K}^{\prime}} \subseteq C_{\text {ヨ\&at }}\left(L_{\mathbf{K}}\right)$. Then $\mathbf{K} \subseteq \mathbf{I S P}(\mathbf{K})^{\text {inj }}$ implies $\mathbf{K}^{\prime} \subseteq \mathbf{I S P}\left(\mathbf{K}^{\prime}\right)^{\text {inj }}$.

Proof. Let $L=L_{\mathbf{K}}$ and $L^{\prime}=L_{\mathbf{K}^{\prime}}$. By Theorem 4 we have $C_{\text {ᄏ\&at }}(L)=C_{\text {\&at }}(L)$, and we must show that $C_{\exists \& a t}\left(L^{\prime}\right) \subseteq C_{\text {\&at }}\left(L^{\prime}\right)$. First, monotonicity yields $C_{\exists \& a t}\left(L^{\prime}\right)$ $\subseteq C_{\exists \text { \&at }}\left(C_{\exists \& \text { at }}(L)\right)$. By idempotence and the sentence above, this last equals $C_{\text {\&at }}(L)$. Finally $C_{\text {\&at }}(L) \subseteq C_{\text {\&at }}\left(C\left(L^{\prime}\right)\right)=C_{\text {\&at }}\left(L^{\prime}\right)$.

REMARK. It is clear from the proof that the condition $L_{\mathbf{K}} \subseteq C\left(L_{\mathbf{K}^{\prime}}\right)$ can be replaced by the more general (but less wieldy) $C_{\& \text { at }}\left(L_{\mathbf{K}}\right) \subseteq C_{\text {\& at }}\left(L_{\mathbf{K}^{\prime}}\right)$.

We close this section by stating a trivial transfer result.

LEMMA 6. Suppose that $\mathbf{K}$ is a regular set of structures, $\mathbf{c}$ is a constant on $K$, and $\mathbf{K}_{\mathbf{c}}$ is the set of expansions of the members of $\mathbf{K}$ to include $\mathbf{c}$.

a) If $\mathbf{K} \subseteq \operatorname{ISP}(\mathbf{K})^{\mathrm{inj}}$ then $\mathbf{K}_{\mathbf{c}} \subseteq \operatorname{ISP}\left(\mathbf{K}_{\mathbf{c}}\right)^{\text {inj }}$.

b) The converse is true if the automorphism group of each member of $\mathbf{K}$ is transitive.

§3. Application to two-element algebras. In this section we prove the claim that $\mathscr{A} \in \operatorname{ISP}(\mathscr{A})^{\mathrm{inj}}$ for every two-element algebra $\mathscr{A}$. Our method is to use Corollary 5 and Lemma 6 to reduce the case of an arbitrary two-element algebra to cases for which the claim is already known to be true. We start with Post's classification of the clones of operations on the set $\mathbf{2}=\{0,1\}$ as presented in [7].

We write $\mathcal{O}_{2}$ for $\mathcal{O}_{\{2\}}$, etc.; $\vee, \wedge, \neg, \mathbf{0}, \mathbf{1}$, are the Boolean operations on $\mathbf{2}$. Define $x \rightarrow y:=(\neg x) \vee y,(x, y, z):=x \wedge(y \vee z), d_{3}(x, y, z):=(x \wedge y) \vee(x \wedge z) \vee(y \wedge z)$, and $\mathrm{RC}(x, y, z):=d_{3}(x, y, \neg z)$. The group operation on 2 with identity 0 is + . For $L$, $L^{\prime} \subseteq \mathcal{O}_{2}$ we write $L \equiv L^{\prime}$ if $L$ and $L^{\prime}$ generate the same clone; that is, $C(L) \cap \mathcal{O}_{2}$ $=C\left(L^{\prime}\right) \cap \mathcal{O}_{2}$.

Lemma 6(a) has a number of obvious applications including, for example, $L_{1}=$ $\{+, \neg\} \equiv\{+, \mathbf{1}\}$ reducing to $\{+\}=L_{3}$ and $C_{3}=\{+, \wedge\} \equiv\{\vee, \wedge, \mathrm{RC}, \mathbf{0}\}$ reducing to $\{\vee, \wedge, \mathrm{RC}\} \equiv C_{4}$. Lemma $6(\mathrm{~b})$ can be applied to subsets of $\left\{d_{3}\right.$, $x+y+z, \neg\}$; for example [1], $D_{2}=\left\{d_{3}\right\}$ reduces to $\left\{d_{3}, \mathbf{0}\right\} \equiv\left\{(x, y, z), d_{3}, \mathbf{0}\right\}=F_{7}^{3}$ and $D_{1}=\left\{d_{3}, x+y+z\right\}$ reduces to $\left\{d_{3}, x+y+z, \mathbf{0}\right\} \equiv\{+, \wedge\}=C_{3}$. Finally, $[1]$ shows that $\{\wedge, \vee\} \subseteq C_{\text {\&at }}(\{\rightarrow\}) \cap C_{\text {\&at }}(\{(x, y, z)\})$ and $\mathrm{RC} \in C_{\text {\&at }}(\{\wedge, \vee\})$, from which we deduce

Lemma 7. For any $L \subseteq C(\{\vee, \wedge, \mathrm{RC}\}), L \cup\{\rightarrow\}$ reduces to $\{\rightarrow\}=F_{4}$ and $L \cup\{(x, y, z)\}$ reduces to $\{(x, y, z)\}=F_{6}$.

Proof. Consider $L \cup\{\rightarrow\}$. We establish the hypothesis of Corollary 5; that is, $\{\rightarrow\} \subseteq C(L \cup\{\rightarrow\})$ and $L \cup\{\rightarrow\} \subseteq C_{\exists \& \text { at }}(\{\rightarrow\})$. It suffices to show that $L \subseteq$ $C_{\exists \& a t}(\{\rightarrow\})$, and indeed

$$
\begin{aligned}
L & \subseteq C(\{\vee, \wedge, \mathrm{RC}\}) \subseteq C\left(C_{\text {\&at }}(\{\vee, \wedge\})\right) \subseteq C\left(C_{\text {\&at }}\left(C_{\text {\&at }}(\{\rightarrow\})\right)\right) \\
& \subseteq\left(C_{\text {ᄏ\&at }}\right)^{3}(\{\rightarrow\})=C_{\text {ヨ\&at }}(\{\rightarrow\}) .
\end{aligned}
$$

The preceding reductions allow us to reduce an arbitrary clone of $\mathrm{O}_{2}$ to one of the following (or a dual): $\varnothing,\{\neg\},\{+\},\{\vee\},\{(x, y, z)\},\{\rightarrow\}$. 
Lemma 8. $\{(x, y, z)\}$ and $\{\rightarrow\}$ reduce to $\{\vee, \wedge\}$.

ProOf. It suffices to reduce $\{(x, y, z)\}$ to $\{\vee, \wedge\}$ and $\{\rightarrow\}$ to $\{\vee, \wedge, 1\}$. We use the remark following Corollary 5. Thus in the first case we must show that $C_{\text {\&at }}(\{\vee, \wedge\}) \subseteq C_{\text {\&at }}(\{(x, y, z)\})$ and $\{(x, y, z)\} \subseteq C_{\exists \& a t}(\{\vee, \wedge\})$. The latter inclusion is immediate. Concerning the former, note that every atomic $\{\vee, \wedge\}$-formula is equivalent (in 2) to a conjunction of formulas of the form

$$
\bigwedge_{i} x_{i} \leq \bigvee_{j} \bigwedge_{k} y_{j k}
$$

Since $\wedge$ is an $\{(x, y, z)\}$-term, it suffices to show that each $x \leq y_{1} \vee \cdots \vee y_{n}$ is definable (in 2) by a \&at $\{(x, y, z)\}$-formula. Indeed for $n \geq 2$

$$
x \leq y_{1} \vee \cdots \vee y_{n} \quad \text { iff } \quad\left(x, y_{1},\left(x, y_{2},\left(\cdots\left(x, y_{n-1}, y_{n}\right) \cdots\right)\right)\right)=x .
$$

The proof for $\{\rightarrow\}$ is similar: $\vee$ and $\mathbf{1}$ are $\{\rightarrow\}$-terms, and

$$
x_{1} \wedge \cdots \wedge x_{n} \leq y \text { iff }\left(x_{1} \rightarrow y\right) \vee \cdots \vee\left(x_{n} \rightarrow y\right)=\mathbf{1} .
$$

We can now prove

THEOREM 9. $\mathscr{A} \in \mathbf{I S P}(\mathscr{A})^{\mathrm{inj}}$ for every two-element algebra $\mathscr{A}$.

Proof. ISP $(\langle\mathbf{2},\{\vee, \wedge\}\rangle)$ is the class of distributive lattices; it is known that the two-element lattice is injective there [3, p. 113]. The situation is the same for $\operatorname{ISP}(\langle\mathbf{2},\{\vee\}\rangle)$, the class of semilattices [4]. $\operatorname{ISP}(\langle\mathbf{2},\{+\}\rangle)$ is the class of vector spaces over the two-element field, and hence every member is injective by elementary linear algebra. The claim can be checked directly for $\operatorname{ISP}(\langle\mathbf{2},\{\neg\}\rangle)$ and for $\operatorname{ISP}(\langle\mathbf{2}, \varnothing\rangle)$. All other cases reduce to these by the previous results of this section.

We conclude this paper with an application to varieties generated by two-element algebras.

COROLLARY 10. Every variety generated by a two-element algebra has EI.

PROOF. Let $\mathscr{A}$ be a two-element algebra and $\mathbf{V}(\mathscr{A})$ the variety it generates. Since $\mathbf{V}(\mathscr{A})$ has, up to isomorphism, only finitely many subdirectly irreducible members [10], it suffices, by Birkhoff's theorem and the first remark of $\S 1$, to show that every maximal subdirectly irreducible member of $\mathbf{V}(\mathscr{A})$ is injective in $\mathbf{V}(\mathscr{A})$.

If $\mathbf{V}(\mathscr{A})$ is simply $\operatorname{ISP}(\mathscr{A})$ together with trivial algebras then the claim follows by Theorem 9. It is known (e.g. [10]) that there are only a handful of exceptions to this situation: those $\mathscr{A}$ whose clones are generated by one of the following sets: $\{\mathbf{0}, \mathbf{1}\}$, $\{\neg\},\{\neg, \mathbf{0}\},\{+, \mathbf{1}\}$, and $\{x+y+z, \neg\}$. The claim can be verified directly in each of these exceptional cases.

\section{REFERENCES}

[1] M. H. Albert, A preservation theorem for ec-structures with applications, this Journal, vol. 52 (1987), pp. 779-785.

[2] P. D. BACSiCh, Amalyamation properties and interpolation theorems for equational theories, Algebra Universalis, vol. 5 (1975), pp. 45-55.

[3] R. Balbes and P. Dwinger, Distributive lattices, University of Missouri Press, Columbia, Missouri, 1974.

[4] G. Bruns and H. LAKSER, Injective hulls of semilattices, Canadian Mathematical Bulletin, vol. 13 (1970), pp. 115-118. 
[5] S. BURRIS and H. WERNER, Sheaf constructions and their elementary properties, Transactions of the American Mathematical Society, vol. 248 (1979), pp. 267-307.

[6] C. C. Chang and H. J. KeISLER, Model theory, North-Holland, Amsterdam, 1973.

[7] R. C. LYNDON, Identities in two-valued calculi, Transactions of the American Mathematical Society, vol. 71 (1951), pp. 457-465.

[8] A. MaCINTYRE, Model completeness, Handbook of mathematical logic (J. Barwise, editor), NorthHolland, Amsterdam, 1977, pp. 139-180.

[9] E. POST, Two-valued iterative systems of mathematical logic, Princeton University Press, Princeton, New Jersey, 1941.

[10] W. TAYLOR, Pure compactifications in quasi-primal varieties, Canadian Journal of Mathematics, vol. 28 (1976), pp. 50-62.

DEPARTMENT OF PURE MATHEMATICS

UNIVERSITY OF WATERLOO

WATERLOO, ONTARIO N2L 3G1, CANADA 